\title{
Super-massive black hole binaries in gas-rich environments
}

\author{
Jorge Cuadra ${ }^{1}$ \\ ${ }^{1}$ Departamento de Astronomía y Astrofísica, Pontificia Universidad Católica de Chile \\ email: jcuadra@astro.puc.cl
}

\begin{abstract}
Black hole binaries form after major galaxy mergers, but their fate is unclear as hardening due to stars gets inefficient at sub-parsec distances. We model an alternative scenario in which the merger is driven by the interaction of the binary with the surrounding gas. $\dagger$
\end{abstract}

Keywords. black hole physics

For the expected masses, the disc will be gravitationally unstable and develop spiral structure, transporting out the angular momentum of the binary and making it shrink. Scaling our results analytically, we find that decay due to gas can be faster than that due to stars for separations below $0.01-0.1 \mathrm{pc}$. The minimum merger time-scale is shorter than the Hubble time for binaries with $M<10^{7} M_{\odot}$ (Cuadra et al. 2009).

The orbit of the binary does not only shrink - it will also become eccentric, tending to $e \approx 0.6$. This is simply due to the instantaneous gravitational interaction of the secondary black hole with the wake it drives in the inner part of the disc. The eccentricity increases the variability of the accretion onto the black holes (Roedig et al. 2011).

For massive discs, cooling conditions could produce their fragmentation and star formation. We are currently investigating the fate of binaries within such systems. Preliminary results show a slower decay rate than in the gaseous case, and an interesting enhancement in the rate of stellar tidal disruptions (Amaro-Seoane, Brem \& Cuadra, ApJ submitted).
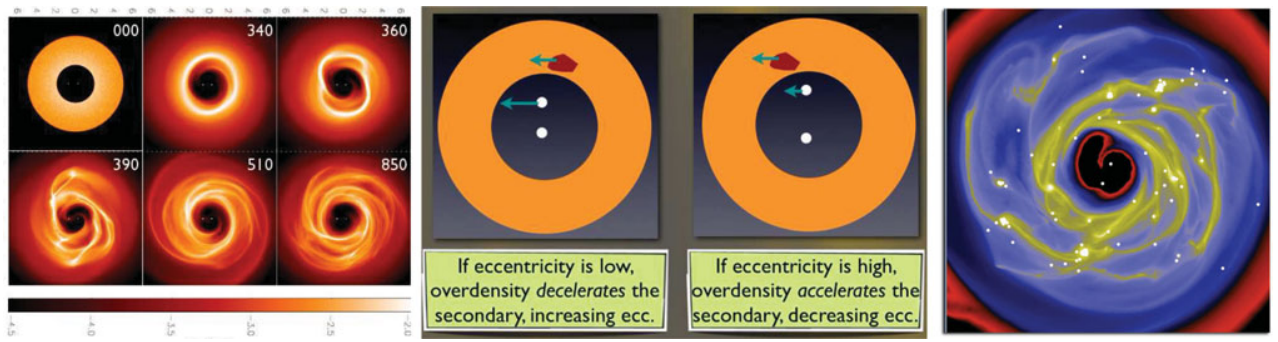

Figure 1. Left: Surface density evolution of the disc around the binary. The spiral pattern that develops transfers out the binary angular momentum. Middle: Binary eccentricity evolution due to the disc. This process makes the binary approach $e \approx 0.6$. Right: Star-forming circumbinary disc. The newly formed stars scatter with the binary, exchanging energy and angular momentum. A relatively large number of stars get tidally disrupted.

\section{References}

Cuadra, J., Armitage, P. J., Alexander, R. D., \& Begelman, M. 2009, MNRAS, 393, 1423

Roedig, C., Dotti, M., Sesana, A., Cuadra, J., \& Colpi, M. 2011, MNRAS, 415, 3033

$\dagger$ This article is based on work done in collaboration with the co-authors of the cited papers. JC acknowledges support from FONDAP (15010003), FONDECYT (11100240), Basal (PFB0609), VRI-PUC (Inicio 16/2010), the FP7 (LACEGAL) and IAU grant GA1090. 